\title{
A DISTRIBUIÇÃO ESPACIAL DAS ESPÉCIES DE ANATIDAE DO CAMPUS II DA UNOESTE, PRESIDENTE PRUDENTE - SP
}

Nome do Autor (a) Principal

LUIZ WALDEMAR DE OLIVEIRA

Nome (s) do Coautor (a) (s)

LÍVIA DONZELLI

DYMIELI DO CARMO CLEMENTE

Instituição ou Empresa

UNOESTE - UNIVERSIDADE DO OESTE PAULISTA - PRESIDENTE PRUDENTE.

E-MAIL: oliveira@unoeste.br

\section{PALAVRAS-CHAVE}

Anatidae. Dendrocygna autumnalis. Cairina moschata.

\section{INTRODUÇÃO}

As aves da família Anatidae possuem hábito aquático, necessitam de águas rasas para se alimentarem bem, a maioria das espécies são migratórias, são nômades, migram a procura de alimento, locais apropriados para nidação, dormitório, muda e até forçadas pelo nível das águas. O Brasil possui poucas espécies de anatídeos, sendo o Estado do Rio Grande do Sul o estado com a maior abundância de espécies (SICK, 2001).

Em áreas onde o estudo da avifauna foi realizado, a presença das espécies da família Anatidade está vinculada à existência de cursos d’água, ou mesmo lagos e lagoas, de acordo com Lopes e Dos Anjos, 2006, que observaram a presença das espécies Dendrocygna viduata e Amazonetta brasiliensis no campus da UEL; Scherer et al, 2006, identificaram as espécies Dendrocygna viduata e Amazonetta 
brasiliensis na zona urbana de Porto Alegre (RS); Pereira e Silva, 2009, identificaram a presença das espécies Dendrocygna autumnalis e Dendrocygna viduata na área urbana de Anápolis-GO; Vogel et al, 2011, encontraram 5 espécies de anatidae no campus da Universidade Estadual de Guarapuava, dentre estas Dendrocygna viduata, Amazonetta brasiliensis e Cairina moschata. As espécies da família Anatidae necessitam de ambientes aquáticos, pois, entre seus itens alimentares constam pequenos invertebrados aquáticos, pequenos crustáceos, pequenos peixes, girinos, vermes e larvas de insetos.

\section{OBJETIVOS}

Analisar a distribuição espacial das espécies de Anatidae identificadas no Campus II da UNOESTE.

\section{METODOLOGIA}

O estudo foi realizado no campus II da UNOESTE, Universidade do Oeste Paulista, município de Presidente Prudente - Sp. Foram eleitas 6 áreas distintas para o estudo, o ribeirão Limoeiro, 5 lagoas distribuídas por diversas áreas do campus e 22 tanques de piscicultura. Os locais de observação (Figura 1) foram assim denominados: Ponto 1 - Lagoa do Biotério; Ponto 2 - Lagoa da Marcenaria; Ponto 3 Lagoa da Horta; Ponto 4 - Lagoa do Reflorestamento; Ponto 5 - ribeirão Limoeiro; e Ponto - Piscicultura. Em cada local, foram estabelecidos pontos fixos de observação (BIBBY, BURGESS, HILL, MUSTOE, 2000). Foram 33 dias de observação, aos sábados, no período de Novembro de 2012 à outubro de 2013, das 7h às 11h da manhã, totalizando $132 \mathrm{~h}$ de esforço amostral, sempre alternando-se o ponto de início de observação. 
Figura 1 - Pontos de observação no Campus II da UNOESTE.

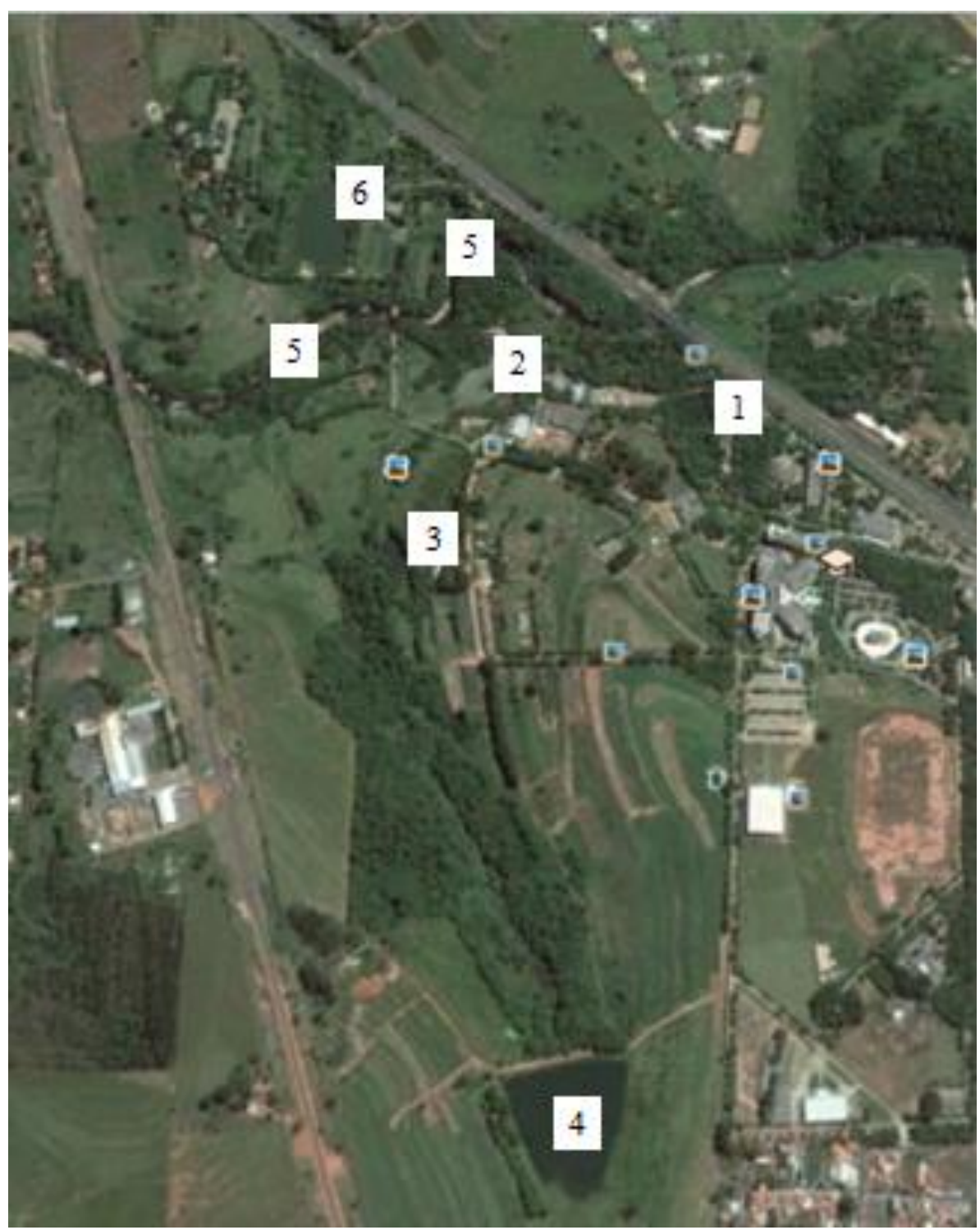

Fonte: Google Eart.

Para a observação das espécies utilizou-se binóculos da marca Nautika ${ }^{\circledR}$ $8 \times 40,120 \mathrm{~m} / 1000 \mathrm{~m}$, para os registros fotográficos dos indivíduos uma câmera fotográfica semi-profissional Fujifilm ${ }^{\circledR}$ Finepix HS10 com 30X Zoom Optico, para a identificação das espécies foram utilizadas obras literárias especializadas (SICK, 2001; ENDRIGO, 2011). 


\section{RESULTADOS E DISCUSSÃO}

Foram observadas no Campus II da UNOESTE, 5 espécies de aves da família Anatidae, duas espécies da subfamília Dendrocygninae: Dendrocygna autumnalis (figura 2.1), e Dendrocygna viduata (figura 2.3), e duas espécies da subfamília Anatinae: Amazonetta brasiliensis (figura 2.2) e Cairina moschata (figura 2.4) e uma espécie exótica, Alopochen aegyptiacus (ganso do Egito). Esta quantidade superior a outros estudos realizados (LOPES; DOS ANJOS, 2006, SCHERER et al, 2006, PEREIRA; SILVA, 2009, VOGEL et al, 2011) pode ser devido á variedade de ambientes aquáticos presentes no campus.

Figura 2: Espécies de aves da família Anatidae encontradas no Campus II da UNOESTE. 1- Dendrocygna autumnalis, 2-Amazonetta brasiliensis, 3- Dendrocygna viduata, 4-Cairina moschata.

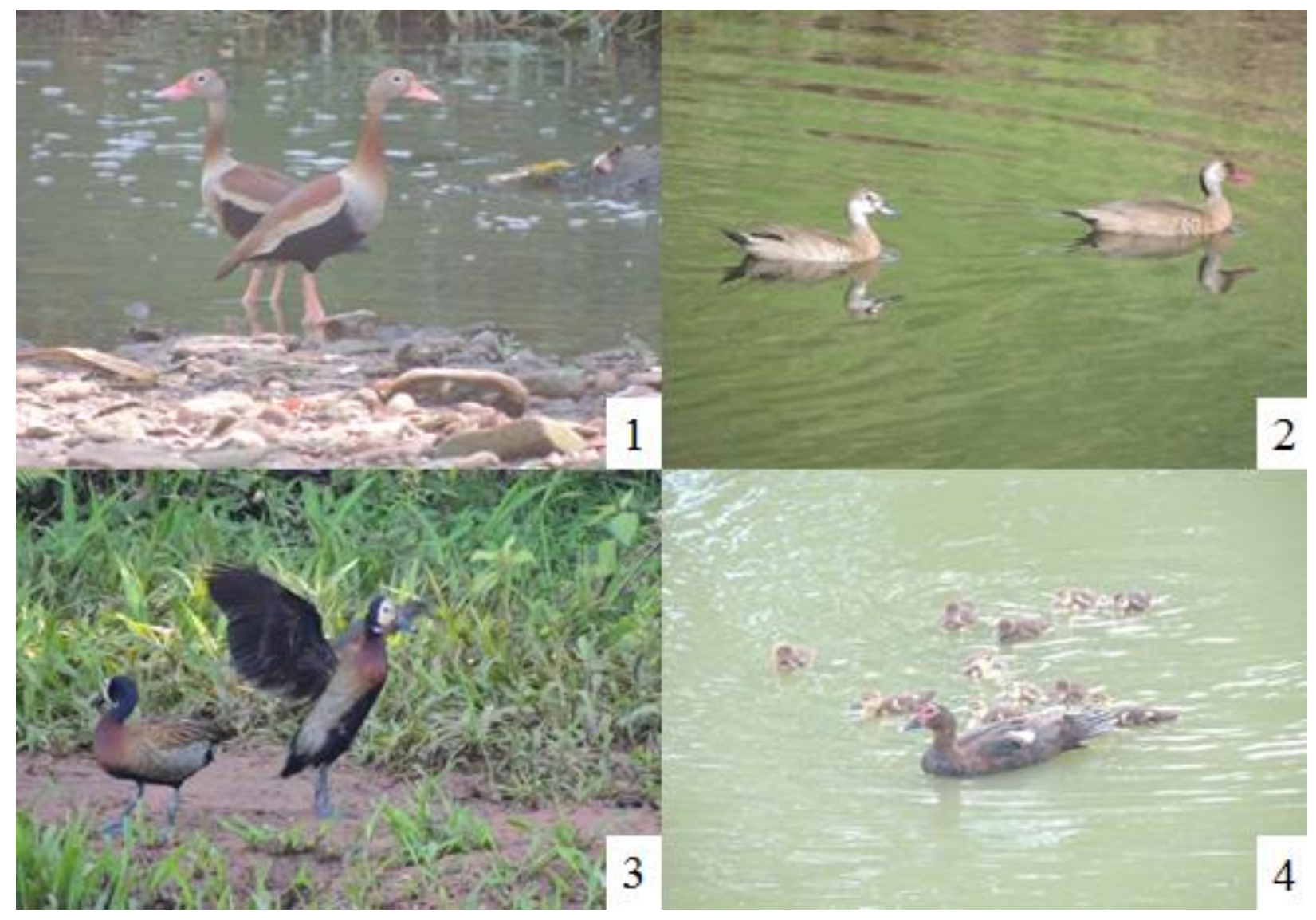

Fonte: Dymyeli do Carmo Clemente, Lívia Donzelli e Luiz Waldemar de Oliveira.

As espécies não ocorreram em todos os pontos de observação (Tabela 1), sendo que a espécie de maior distribuição nos pontos estudados foi Cairina 
moschata e as de menor distribuição foram Dendrocygna autumnalis e Alopochen aegyptiacus.

Tabela 1: Distribuição das espécies da família Anatidae por pontos de observação.

\begin{tabular}{ccccccc}
\hline \multirow{2}{*}{ Espécies } & \multicolumn{5}{c}{ Pontos de observação } \\
\cline { 2 - 6 } & Ponto 1 & Ponto 2 & Ponto 3 & Ponto 4 & Ponto 5 & Ponto 6 \\
\hline Amazonetta brasiliensis & & & $\mathrm{X}$ & $\mathrm{X}$ & $\mathrm{X}$ & $\mathrm{X}$ \\
Cairina moschata & $\mathrm{X}$ & $\mathrm{X}$ & $\mathrm{X}$ & & $\mathrm{X}$ & $\mathrm{X}$ \\
Dendrocygna autumnalis & & & & & $\mathrm{X}$ \\
Dendrocygna viduata & & $\mathrm{X}$ & $\mathrm{X}$ & \\
Alopochen aegyptiacus & & & & $\mathrm{X}$ \\
\hline
\end{tabular}

Com relação à frequência (Tabela 2), Cairina moschata foi a única espécie que esteve presente no campus em 100\% das visitas, Amazonetta brasiliensis esteve presente em $81 \%$ das visitas. Alopochen aegyptiacus, espécie exótica africana, foi observado no campus por um período contínuo de 4 meses, um só indivíduo, sempre restrito á margem norte do córrego limoeiro.

Tabela 2: Frequência das espécies da família Anatidae por pontos de observação e frequência total (F.total).

\begin{tabular}{ccccccccc}
\hline Espécies & \multicolumn{7}{c}{ Pontos de observação } \\
\cline { 2 - 8 } & Ponto & Ponto & Ponto & Ponto & Ponto & Ponto & F. \\
& 1 & 2 & 3 & 4 & 5 & 6 & total \\
\hline $\begin{array}{c}\text { Amazonetta brasiliensis } \\
\text { Cairina moschata }\end{array}$ & 0 & 0 & $6 \%$ & $9 \%$ & $69 \%$ & $9 \%$ & $81 \%$ \\
$\begin{array}{c}\text { Dendrocygna } \\
\text { autumnalis }\end{array}$ & 0 & 0 & 0 & 0 & $9 \%$ & 0 & $9 \%$ \\
$\begin{array}{c}\text { Dendrocygna viduata } \\
\text { Alopochen aegyptiacus }\end{array}$ & 0 & 0 & $9 \%$ & $6 \%$ & 0 & 0 & $12 \%$ \\
& 0 & 0 & 0 & 0 & $33 \%$ & 0 & $33 \%$
\end{tabular}

De acordo com a frequência (VIELLIARD; SILVA, 1990), as espécies consideradas residentes são Cairina moschata e Amazonetta brasiliensis, espécies estas que, inclusive, utilizam a área do campus para procriar e cuidar de sua prole. 
A alta frequência de Amazonetta brasiliensis no Ribeirão Limoeiro (ponto 5) se deve ao seu hábito alimentar e estratégia de reprodução, assim como a presença mais acentuada de Cairina moschata nos pontos 1,2 e menor, mas também relevante no ponto 5. A ausência de espécies na lagoa do reflorestamento (ponto 4) se deve provavelmente por ser uma área mais exposta a predadores e inadequada para nidação, não sendo utilizada por nenhuma espécie em estudo para procriação ou moradia. As demais espécies apenas usaram a área do campus para repouso ou alimentação.

\section{CONCLUSÃO}

Cairina moschata é a espécie de maior distribuição espacial no Campus II da UNOESTE, seguida de Amazonetta brasiliensis.

Cairina moschata e Amazonetta brasiliensis são espécies residentes do campus II da UNOESTE, apresentando a maior frequência com relação aos dias visitados.

\section{REFERÊNCIAS}

BIBBY, C.J.; BURGESS, N.D.; HILL, D.A.; MUSTOE, S.H. Bird census techniques. $2^{\mathrm{a}}$ ed. London: Academic Press, 2000.

ENDRIGO, E. Aves: Estado de São Paulo: aves \& fotos. São Paulo, 2010.

LOPES, E. V.; ANJOS, L. A composição da avifauna da Universidade Estadual de Londrina, Norte do Paraná, Brasil. Revista Brasileira de Zoologia, v. 23, n. 1, p. 145-156, 2006.

PEREIRA, K.D.L.; SILVA, R. Levantamento da avifauna da área urbana de Anápolis, Goiás. Ensaios e Ciências: Ciências Biológicas, Agrárias e da Saúde. V13, n 2, p 33-46, 2009.

SCHERER, J.F.M.; SCHERER, A.L.; PETRY, M.V.; TEIXEIRA, E.C. Estudo da avifauna associada à área úmida situada no Parque Mascarenhas de Moraes, zona urbana de Porto Alegre (RS).

Biotemas, V. 19, N. 1, p. 107-110, 2006.

SICK, H. Ornitologia Brasileira. Rio de Janeiro, Nova Fronteira, 2001.

VIELLIARD, J. M. E. \& SILVA, W. R. Nova metodologia de levantamento quantitativo e primeiros resultados no interior do Estado de São Paulo. Pp. 117-151. In: ENCONTRO NACIONAL DE ANILHADORES DE AVES, Recife. Anais do IV Enave. Recife: Universidade Federal de Pernambuco. 1990.

VOGEL, H.F.; METRI, R.; ZAWADZKI, C.H.; MOURA, M.O. Avifauna from a campus of Universidade Estadual do Centro-Oeste,Guarapuava, Paraná State, Brazil. Acta Scientiarum. Biological Sciences, Maringá, v. 33, n. 2, p. 197-207, 2011. 